\title{
ON THE $\sigma$-CLASS GENERATED BY OPEN BALLS
}

\author{
STEVE JACKSON AND R. DANIEL MAULDIN ${ }^{1}$
}

\begin{abstract}
We show that in the finite dimensional space $\mathbb{R}^{d}$ provided with a metric induced by a norm, the collection of Borel sets is the smallest collection containing the open balls and closed under complements and countable disjoint unions.
\end{abstract}

\section{INTRODUCTION}

A family $\mathcal{L}$ of subsets of a set $X$ is said to be a $\sigma$-class provided $\mathcal{L}$ is closed under complements and countable disjoint unions. Such families arise in the study of quantum logic and have been termed a concrete quantum logic [6] or a q-sigmaalgebra [7]. If $\mathcal{C}$ is a family of subsets of $X$, then $\mathcal{L}(\mathcal{C})$, the $\sigma$-class generated by $\mathcal{C}$, is the smallest $\sigma$-class which includes $\mathcal{C}$. It is known that if $\mathcal{C}$ is closed with respect to intersection, then $\mathcal{L}(\mathcal{C})=\mathcal{B}(\mathcal{C})$, the Borel field or $\sigma$-algebra generated by $\mathcal{C}$. In this paper, $X$ is a metric space and we concern ourselves with the family $\mathcal{L}_{0}=\mathcal{L}_{0}(X)$, the $\sigma$-class generated by the family of all open balls in $X$. Since a $\sigma$-class is closed under the intersection of a descending sequence of sets [6], $\mathcal{L}_{0}=\mathcal{B}_{0}$, the Borel field generated by the open subsets of $X$, provided every open set is in $\mathcal{L}_{0}$. The question naturally arises as to whether $\mathcal{L}_{0}$ is always $B_{0}$ for a metric space $X$. The answer to this question is no even for compact metric spaces. Davies has given an example of a compact metric space, $D$ and two distinct Borel probability measures $\mu$ and $\nu$ which agree on all balls [3]. Of course, if two measures give the same measure to all balls, then they agree on $\mathcal{L}_{0}$. Thus, for Davies' space, $\mathcal{L}_{0} \neq \mathcal{B}_{0}$. On the other hand, it is clear that if $X=\mathbb{R}^{1}$, then $\mathcal{L}_{0}=\mathcal{B}_{0}$. In fact, Olejček showed that if $X=\mathbb{R}^{2}$, then $\mathcal{L}_{0}\left(\mathbb{R}^{2}\right)=\mathcal{B}\left(\mathbb{R}^{2}\right)$ [7]. Also, it is known that if two Borel probability measures, $\mu$ and $\nu$ agree on the family of all balls in a separable Banach space, then $\mu=\nu$ [9]. The technique of proof uses Fourier transforms and does not directly address our problem. However, this does provide some evidence that the answer to the following question (which seems to have been posed in some form by Preiss and independently by Neubrunn [6]) is positive.

QUESTION. Let X be a separable Banach space. Is it true that the $\sigma$-class generated by the family of all open balls in $X$ is the standard Borel field on $X$ ?

In this paper, we shall show that the answer is yes for finite dimensional Banach spaces, by proving the following theorem in $\S 3$.

Theorem 1.1. For each positive integer $d$, consider $\mathbb{R}^{d}$ with a metric induced by some norm. Then $\mathcal{L}_{0}\left(\mathbb{R}^{d}\right)=\mathcal{B}_{0}\left(\mathbb{R}^{d}\right)$.

We shall prove the theorem in $\S 2$ for the special case that the norm is the Euclidean norm. The proof in this case requires a slight variation of Besicovitch's

Key words and phrases. Borel sets, Besicovitch covering.

${ }^{1}$ Research supported by NSF Grant DMS-9502952. 
covering theorem. In $\S 3$ we give the necassary changes to the proof for a metric induced by a general norm. It turns out that not only do we need the variation of the covering theorem, but also a result on the Hausdorff measure of the intersection of the boundaries of random translates of bounded, open, convex sets.

\section{The Euclidean Case}

As we remarked above, $\mathcal{L}_{0}$ is closed under countable increasing unions and countable decreasing intersections.

Our proofs make use of the following combinatorial lemma, valid for any metric space. In essence, the lemma states that if there are $p$ nets of open balls in a metric space $X$ such that a closed set $Q$ can be covered by the level $j$ sets in the nets, then $Q \in \mathcal{L}_{0}(X)$.

Lemma 2.1. Let $(X, \rho)$ be a metric space, and $Q \subseteq X$ be closed. Suppose there are finitely many families $\mathcal{N}^{1}, \ldots, \mathcal{N}^{p}$ of open balls in $X$, with each $\mathcal{N}^{i}=\cup_{j} \mathcal{N}_{j}^{i}$, satisfying the following properties.

1. For every ball $B \in \mathcal{N}_{j}^{i}, \operatorname{diam}(B)<1 / j$.

2. For every $j, Q \subseteq \bigcup_{1 \leq i \leq p} \cup \mathcal{N}_{j}^{i}$. That is, the collection of balls $\bigcup_{1 \leq i \leq p} \mathcal{N}_{j}^{i}$ covers $Q$.

3. For any $1 \leq i \leq p$, if $B_{1}, B_{2} \in \mathcal{N}^{i}$, then either $B_{1} \cap B_{2}=\emptyset, B_{1} \subseteq B_{2}$, or $B_{2} \subseteq B_{1}$.

Then $Q \in \mathcal{L}_{0}(X)$.

Proof. Define a sequence of sets $G_{1}, G_{2}, \ldots, G_{p}$ as follows. Let

$$
G_{1}=\bigcap_{k} \bigcup_{n \geq k} E_{n}
$$

where

$$
E_{n}=\bigcup\left\{B \in \mathcal{N}_{n}^{1}: B \cap Q \neq \emptyset\right\}
$$

Next, let

$$
G_{2}=\bigcup_{i_{1}} \bigcap_{k \geq i_{1}} \bigcup_{n \geq k} E_{n, i_{1}}
$$

where

$$
E_{n, i_{1}}=\bigcup\left\{B \in \mathcal{N}_{n}^{2}: B \cap Q \neq \emptyset \wedge B \nsubseteq A \text { for any } A \in \bigcup_{s=i_{1}}^{n} \mathcal{N}_{s}^{1}\right\}
$$

In general, for $t \leq p$, define

$$
G_{t}=\bigcup_{i_{1}} \bigcup_{i_{2} \geq i_{1}} \cdots \bigcup_{i_{t-1} \geq i_{t-2}} \bigcap_{k \geq i_{t-1}} \bigcup_{n \geq k} E_{n, i_{1}, \ldots, i_{t-1}},
$$

where 


$$
\begin{aligned}
E_{n, i_{1}, \ldots, i_{t-1}}=\bigcup\left\{B \in \mathcal{N}_{n}^{t}: B \cap Q \neq \emptyset \wedge B \nsubseteq A \text { for any } A \in \bigcup_{s=i_{1}}^{n} \mathcal{N}_{s}^{1} \cup\right. \\
\left.\qquad \bigcup_{s=i_{2}}^{n} \mathcal{N}_{s}^{2} \cup \cdots \cup \bigcup_{s=i_{t-1}}^{n} \mathcal{N}_{s}^{t-1}\right\} .
\end{aligned}
$$

To finish the proof, it suffices to show that the sets $G_{t}$ have the following properties.

(i) For each $t, G_{t} \in \mathcal{L}_{0}$.

(ii) The sets $G_{t}, 1 \leq t \leq p$ are pairwise disjoint.

(iii) $Q=\cup_{t=1}^{p} G_{t}$.

First we note the following two properties.

(A). If $\mathcal{H} \subset \mathcal{N}^{t}$ for some $1 \leq t \leq p$, then $\cup \mathcal{H} \in \mathcal{L}_{0}$. and

(B). For each $1 \leq t \leq p, j \leq t-1$, and $i_{j} \leq i_{j}^{\prime}$ we have

$$
E_{n, i_{1}, \ldots, i_{j}, \ldots, i_{t-1}} \subset E_{n, i_{1}, \ldots, i_{j}^{\prime}, \ldots, i_{t-1}}
$$

i.e., the sets $\mathrm{E}$ are increasing in the $j^{t h}$ variable.

Property (A) follows immediately from property 3 of the nets $\mathcal{N}^{t}$, while property (B) is immediate from equation (2).

We first verify (i). For each $i_{1}, \ldots, i_{t-1}$ and $k \geq i_{t-1}$,

$$
\bigcup_{n \geq k} E_{n, i_{1}, \ldots, i_{t-1}} \in \mathcal{L}_{0}
$$

by property (A). For each $i_{1}, \ldots, i_{t-1}$ and $k \leq k^{\prime}$ we also have

$$
\bigcup_{n \geq k^{\prime}} E_{n, i_{1}, \ldots, i_{t-1}} \subseteq \bigcup_{n \geq k} E_{n, i_{1}, \ldots, i_{t-1}}
$$

Thus, for fixed $i_{1}, \ldots, i_{t-1}$, the set $\cap_{k \geq i_{t-1}} \cup_{n \geq k} E_{n, i_{1}, \ldots, i_{t-1}}$ is a decreasing intersection of sets in $\mathcal{L}_{0}$, and thus lies in $\mathcal{L}_{0}$.

Fix $i_{1}, \ldots, i_{j-1}$, where $j<t$. We claim the sets

$$
S_{i_{j}}=S_{i_{j}}\left(i_{1}, \ldots, i_{j-1}\right)=\bigcup_{i_{j+1} \geq i_{j}} \ldots \bigcup_{i_{t-1} \geq i_{t-2}} \bigcap_{k \geq i_{t-1}} \bigcup_{n \geq k} E_{n, i_{1}, \ldots, i_{j}, \ldots, i_{t-1}}
$$

are increasing with $i_{j}$. To see this, let $i_{j} \leq i_{j^{\prime}}$. Suppose $x \in S_{i_{j}}$. This means there are integers $i_{j} \leq i_{j+1} \leq \ldots \leq i_{t-1}$ such that

$$
x \in \bigcap_{k \geq i_{t-1}} \bigcup_{n \geq k} E_{n, i_{1}, \ldots, i_{j-1}, i_{j}, i_{j+1}, \ldots, i_{t-1}} .
$$

That is, for infinitely many $n, x \in E_{n, i_{1}, \ldots, i_{j-1}, i_{j}, i_{j+1}, \ldots, i_{t-1}}$. But, by property (B), we then have for infinitely many $n$, that $x \in E_{n, i_{1}, \ldots, i_{j-1}, i_{i}^{\prime}, i_{j+1}^{\prime}, \ldots, i_{t-1}^{\prime}}$ where for $l>j, i_{l}^{\prime}=\max \left\{i_{l}, i_{j}^{\prime}\right\}$. Thus $x \in S_{i_{j}^{\prime}}$. An immediate induction now gives $G_{t} \in \mathcal{L}_{0}$.

Next we verify (ii). Suppose $x \in G_{t_{1}} \cap G_{t_{2}}$ with $t_{1}<t_{2}$. Fix $i_{1}^{\prime} \leq i_{2}^{\prime} \leq \ldots \leq i_{t_{1}-1}^{\prime}$ such that $x \in \cap_{k \geq i_{t_{1}-1}^{\prime}} \cup_{n \geq k} E_{n, i_{1}^{\prime}, \ldots, i_{t_{1}-1}^{\prime}}$. Also, fix $i_{1}^{\prime \prime} \leq i_{2}^{\prime \prime} \leq \ldots \leq i_{t_{2}-1}^{\prime \prime}$ such that $x \in \cap_{k \geq i_{t_{2}-1}^{\prime \prime}} \cup_{n \geq k} E_{n, i_{1}^{\prime \prime}, \ldots, i_{t_{2}-1}^{\prime \prime}}$. Let $i_{j}=\max \left\{i_{j}^{\prime}, i_{j}^{\prime \prime}\right\}$ for $j \leq t_{1}-1$ and $i_{j}=\max \left\{i_{j}^{\prime \prime}, i_{t_{1}-1}^{\prime}\right\}$ for $j>t_{1}-1$. Thus $x \in \cap_{k \geq i_{t_{1}-1}} \cup_{n \geq k} E_{n, i_{1}, \ldots, i_{t_{1}-1}}$ and 
$x \in \cap_{k \geq i_{t_{2}-1}} \cup_{n \geq k} E_{n, i_{1}, \ldots, i_{t_{1}-1}, \ldots, i_{t_{2}-1}}$. In particular, $x \in E_{n, i_{1}, \ldots, i_{t_{1}-1}}$, for some $n \geq i_{t_{1}}$. Fix a ball $B=B_{\rho}(z, \epsilon)$ in $\mathcal{N}_{n}^{t_{1}}$ such that $x \in B$. Now, let $m>n$ with $x \in E_{m, i_{1}, \ldots, i_{t_{2}-1}}$. So, there is some ball $C$ such that $x \in C \in \mathcal{N}_{m}^{t_{2}}$ and $C \nsubseteq A$ for any ball $A \in \cup_{s=i_{t_{1}}}^{m} \mathcal{N}_{s}^{t_{1}}$. Since $i_{t_{1}} \leq n \leq m$, we have $C \nsubseteq B$. Thus, $\operatorname{diam} C \geq \epsilon-\rho(x, z)$. But, $\operatorname{diam}(C)<1 / m$ as $C \in \mathcal{N}_{m}^{t_{2}}$. Taking $m$ sufficiently large, we have a contradiction.

Finally, we verify (iii). As $Q$ is closed, clearly $G_{t} \subseteq Q$ for all $1 \leq t \leq p$. To see the other inclusion, let $x \in Q$. Now, $\forall n \exists i \leq p\left[x \in \cup \mathcal{N}_{n}^{i}\right]$ by property 2 of the nets. Thus, there is a least $t$ such that for infinitely many $n, x$ lies in some ball in $\mathcal{N}_{n}^{t}$. If $t=1$, then clearly $x \in G_{1}$. Otherwise, fix $i_{1} \leq \ldots \leq i_{t-1}$ such that $\forall n \geq i_{l}, x$ lies in no ball in $\mathcal{N}_{n}^{l}$. However, there are infinitely many $n \geq i_{t-1}$ such that $x$ lies some ball $B_{n} \in \mathcal{N}_{n}^{t}$, and we cannot have $B_{n} \subseteq B$ for any ball $B \in \mathcal{N}_{m}^{l}$ for $l<t, m \geq i_{l}$. Thus, $x \in \cap_{k \geq i_{t-1}} \cup_{n \geq k} E_{n, i_{1}, \ldots, i_{t-1}}$, and so $x \in G_{t}$.

We now specialize to the case $X=\mathbb{R}^{d}$ with the Euclidean distance. To complete the proof of theorem 1.1 for this case, it suffices to show that every compact $Q \subseteq \mathbb{R}^{d}$ is in $\mathcal{L}_{0}$, since every closed set in $\mathbb{R}^{d}$ is an increasing union of compact sets. So, fix a compact set $Q \subseteq \mathbb{R}^{d}$. It now suffices to construct finitely many families of balls $\mathcal{N}^{1}, \ldots, \mathcal{N}^{p}$ satisfying the hypotheses of lemma 2.1 . We use the following slight variation of a theorem of Besicovitch [1].

Theorem 2.1 (Besicovitch). For every integer $d \geq 1$, there is an integer $b(d)$ (depending only on d) with the following properties. Let $A$ be a bounded subset of $\mathbb{R}^{d}$, and $\mathcal{B}$ a family of open balls (in the usual Euclidean metric) such that each point of $A$ is the center of some ball in $\mathcal{B}$. Then there are sub-families $\mathcal{C}_{1}, \ldots, \mathcal{C}_{b(d)} \subseteq \mathcal{B}$ with $A \subseteq \bigcup_{i=1}^{b(d)} \cup \mathcal{C}_{i}$ such that $\forall i \forall B_{1}, B_{2} \in \mathcal{C}_{i} \bar{B}_{1} \cap \bar{B}_{2}=\emptyset$.

Remark 2.1. We have not found Besicovitch's covering theorem stated exactly in this form in the literature. However, a proof can be given by following the proof in [4]. One simply replaces the closed balls in the proof with our open balls and also in the last stage the families $\mathcal{B}_{1}, \mathcal{B}_{2}, \ldots$ are constructed inductively by adding a new ball to the collection not when it is disjoint from the preceding balls, but rather when its closure is disjoint from the closures of the preceding balls.

Remark 2.2. The proof below really only uses the special case of the Besicovitch theorem where all the balls in $\mathcal{B}$ have the same radius. In this case, the theorem is comparatively easy.

Definition 2.1. If $\mathcal{B}$ is a family of balls in $\mathbb{R}^{d}$, we say $x$ is a multiple point of $\mathcal{B}$ if $x$ lies on the boundaries of at least $d$ of the balls in $\mathcal{B}$. Also, for $x \in \mathbb{R}^{d}$ we define the multiplicity of $x$ with respect to $\mathcal{B}, \operatorname{mult}_{\mathcal{B}}(x)$, to be the number of balls $B \in \mathcal{B}$ such that $x \in \partial(B)$.

We note the simple fact that if $d$ balls in $\mathbb{R}^{d}$ meet in more than two points, then the centers of these balls lie on a $d-2$ dimensional plane in $\mathbb{R}^{d}$. We say a set of points in $\mathbb{R}^{d}$ is in general position if no $d$ of the points lie on a $d-2$ dimensional plane in $\mathbb{R}^{d}$ (for $d=2$ this imposes no restriction). Thus, if the centers of a finite family of balls are in general position, then there are only finitely many multiple points for this family.

Let $p=b(d)(d+2)$. We define the finite sub-families of balls $\mathcal{N}_{j}^{i}$, for $1 \leq i \leq p$, inductively on $j$. To begin, let $\mathcal{B}=\{B(x, 1): x \in Q\}$. Applying theorem 2.1, 
there are finite sub-families $\mathcal{C}^{1}, \ldots, \mathcal{C}^{b(d)} \subseteq \mathcal{B}$ such that $\left(\right.$ a) $\mathcal{C} \doteq \bigcup_{i=1}^{b(d)} \mathcal{C}^{i}$ covers $Q$ and (b) the closures of the balls in each $\mathcal{C}^{i}$ form a pairwise disjoint collection. By compactness, we may further assume that each $\mathcal{C}^{i}$ is finite. Also by compactness, there is an $\epsilon>0$ such that if each ball in $\mathcal{C}$ is translated by no more than $\epsilon$, thereby producing new families $\tilde{\mathcal{C}}=\tilde{\mathcal{C}}^{1} \cup \cdots \cup \tilde{\mathcal{C}}^{b(d)}$, then these new families also satisfy (a), (b). Enumerate the balls in $\mathcal{C}$ as $C_{1}, \ldots, C_{t}$. By successively translating each ball in $\mathcal{C}$ by less than $\epsilon$, it is now easy to arrange that the centers of the balls in $\tilde{\mathcal{C}}$ are in general position, and $\partial\left(\tilde{C}_{k}\right)$ does not contain any of the multiple points relative to $\tilde{C}_{1}, \ldots, \tilde{C}_{k-1}$. Having done this, we let $\mathcal{N}_{1}^{1}=\tilde{\mathcal{C}}^{1}, \ldots, \mathcal{N}_{1}^{b(d)}=\tilde{\mathcal{C}}^{b(d)}$, and $\mathcal{N}_{1}^{i}=\emptyset$ for $b(d)<i \leq p$.

Assume now $\mathcal{N}_{j}^{i}$ has been defined for $1 \leq i \leq p$ and $j \leq n$. Let $\mathcal{D}=\bigcup_{i=1}^{p} \bigcup_{j=1}^{n} \mathcal{N}_{j}^{i}$ be the finite collection of balls so far defined. Assume these families satisfy properties 1, 2 of lemma 2.1, the following slight strengthening $\left(3^{\prime}\right)$ of (3), and two extra properties:

$3^{\prime}$. For any $1 \leq i \leq p$ and distinct balls $B_{1}, B_{2} \in \bigcup_{j=1}^{n} \mathcal{N}_{j}^{i}, \partial\left(\bar{B}_{1}\right) \cap \partial\left(\bar{B}_{2}\right)=\emptyset$.

4. The centers of the balls in $\mathcal{D}$ are in general position.

5. $\forall x \in \mathbb{R}^{d} \operatorname{mult}_{\mathcal{D}}(x) \leq d$.

For $n=1$, the $\mathcal{N}_{1}^{i}$ satisfy the required properties.

Let $\partial(\mathcal{D})=\bigcup\{\partial(B): B \in \mathcal{D}\}$ be the union of the boundaries of all the balls so far constructed. For each $x \in \partial(\mathcal{D})$, let $B_{x}$ be a ball centered at $x$ of radius $<\frac{1}{n+1}$ such that $K_{x} \doteq\left\{i: \bar{B}_{x} \cap \partial(B) \neq \emptyset\right.$ for some $\left.B \in \cup_{j \leq n} \mathcal{N}_{j}^{i}\right\}$ has size $\leq d$. This is possible since $x$ lies on at most $d$ boundaries of balls in $\mathcal{D}$. Let $\mathcal{B}=\left\{B_{x}: x \in \partial(\mathcal{D})\right\}$. Apply now theorem 2.1 to the set $\partial(\mathcal{D})$ and the balls $\mathcal{B}$. This produces finite sub-families $\mathcal{W}^{1}, \ldots, \mathcal{W}^{b(d)} \subseteq \mathcal{B}$ whose union $\mathcal{W}$ covers $\partial(\mathcal{D})$ such that the closures of any two distinct balls in any $\mathcal{W}^{i}$ are disjoint. We now "color" the balls in $\mathcal{W}$, that is, we decide for which $i$ we shall place the ball in $\mathcal{N}_{n+1}^{i}$. For any ball $B_{x} \in \mathcal{W}_{1}$, color the ball the least $i$ not in $K_{x}$. Note that the color of each ball in $\mathcal{W}^{1}$ is an integer $\leq d+1$. In general, suppose we have colored the balls in $\mathcal{W}^{1}, \ldots, \mathcal{W}^{k}$, using only colors $\leq k(d+1)$. For each ball $B_{x} \in \mathcal{W}^{k+1}$, color it the least integer $i>k(d+1)$ not in $K_{x}$. Since $\left|K_{x}\right| \leq d$, each ball in $\mathcal{W}^{k+1}$ gets a color $\leq(k+1)(d+1)$. Continuing, we color all the balls in $\mathcal{W}$, using at most $b(d)(d+1)$ colors. By construction, for any two balls $B_{1}, B_{2} \in \mathcal{W} \cup \mathcal{D}$, if $\partial\left(B_{1}\right) \cap \partial\left(B_{2}\right) \neq \emptyset$, then $B_{1}, B_{2}$ have different colors.

Consider now $K=Q-\bigcup\{B: B \in \mathcal{W}\}$. Let $0<\delta<\min \{1 /(n+1), \rho(K, \partial(\mathcal{D}))\}$. Let $\mathcal{C}=\{B(x, \delta): x \in K\}$. Applying theorem 2.1 to $K$ and $\mathcal{C}$ produces families $\mathcal{V}=\mathcal{V}^{1} \cup \cdots \cup \mathcal{V}^{b(d)} \subseteq \mathcal{C}$ such that $\mathcal{V}$ covers $K$ and the closures of any two distinct balls in any $\mathcal{V}^{i}$ are disjoint. Color now all the balls in $\mathcal{V}^{k}$ color $b(d)(d+1)+k$. Thus, we use colors $\leq b(d)(d+2)=p$ in coloring the balls from $\mathcal{W} \cup \mathcal{V}$.

By construction, if $B_{1}, B_{2} \in \mathcal{D} \cup \mathcal{W} \cup \mathcal{V}$ have the same color, then $\partial\left(B_{1}\right) \cap \partial\left(B_{2}\right)=$ $\emptyset$. Also, the balls in $\mathcal{W} \cup \mathcal{V}$ cover $Q$. By compactness, there is an $\epsilon>0$ such that if all the balls in $\mathcal{W} \cup \mathcal{V}$ are translated no more than $\epsilon$, then the resulting families $\tilde{\mathcal{W}}, \tilde{\mathcal{V}}$ also have these two properties. We successively translate the balls in $\mathcal{W} \cup \mathcal{V}$, producing $\tilde{\mathcal{W}}, \tilde{\mathcal{V}}$, so that the collection of centers of the balls in $\mathcal{F}=\mathcal{D} \cup \mathcal{W} \cup \mathcal{V}$ is in general position, and $\forall x$ mult $_{\mathcal{F}}(x) \leq d$.

For $1 \leq i \leq p$, let $\mathcal{N}_{n+1}^{i}$ be all the balls in $\tilde{\mathcal{W}} \cup \tilde{\mathcal{V}}$ of color $i$. The $\mathcal{N}_{n+1}^{i}$ then satisfy (1), (2), $\left(3^{\prime}\right),(4)$, and (5) as required. This completes the proof of theorem 1.1 for the Euclidean metric on $\mathbb{R}^{d}$. 


\section{General Norms on $\mathbb{R}^{d}$}

We turn now to the general case of theorem 1.1. Fix a norm $x \rightarrow\|x\| \in \mathbb{R}^{\geq 0}$ on $\mathbb{R}^{d}$, and let $\rho(x, y)=\|x-y\|$ be the corresponding metric. Any norm on $\mathbb{R}^{d}$ is necessarily continuous, so the balls $B_{\rho}(x, r)$ are open, convex, bounded sets containing $x$, which are also symmetric about $x$ (recall that in any topological vector space $V$ the continuous norms are in correspondence with the open, convex, symmetric neighborhoods $U$ of $\mathbf{0}$ which are bounded in the sense that for all $x \neq$ $\mathbf{0}, \sup \{t: t x \in U\}<\infty)$. Throughout this section, $\mathcal{H}^{s}$ refers to $s$-dimensional Hausdorff measure. When we say "for almost all $y \in \mathbb{R}^{d}$," we mean with respect to Lebesgue measure on $\mathbb{R}^{d}$, that is $\mathcal{H}^{d}$.

Inspecting the proof of theorem 1.1 shows that there are two properties of the family of Euclidean balls in $\mathbb{R}^{d}$ which we must generalize to the balls $B_{\rho}(x, r)$. First is the Besicovitch theorem, and secondly we must generalize the "general position" argument used in that proof.

For the first, we use the following variation of Besicovitch's theorem. This is a slightly altered form of the theorem proven by A. P. Morse [5].

Theorem 3.1 (Morse). For every integer $d \geq 1$ and metric on $\mathbb{R}^{d}$ induced by some norm, there is an integer $b(d)$ (depending only on $d$ and the norm) with the following properties. Let $A$ be a bounded subset of $\mathbb{R}^{d}$, and $\mathcal{B}$ a family of open balls (with respect to the metric) such that each point of $A$ is the center of some ball in $\mathcal{B}$. Then there are sub-families $\mathcal{C}_{1}, \ldots, \mathcal{C}_{b(d)} \subseteq \mathcal{B}$ with $A \subseteq \bigcup_{i=1}^{b(d)} \cup \mathcal{C}_{i}$ such that $\forall i$ $\forall B_{1}, B_{2} \in \mathcal{C}_{i} \bar{B}_{1} \cap \bar{B}_{2}=\emptyset$.

Remark 3.1. Again, we have not found Morse's covering theorem stated exactly in this form in the literature. However, again a proof can be given by following the proof in [4]. One simply replaces the closed balls in the proof with our open balls (with respect to the norm). Again, in the last stage the families $\mathcal{B}_{1}, \mathcal{B}_{2}, \ldots$ are constructed inductively by adding a new ball to the collection not when it is disjoint from the preceding balls, but rather when its closure is disjoint from the closures of the preceding balls. Finally, one also also uses the fact that there is an integer $J(d)$ depending only on $d$ and the norm such that if $\mathcal{F}$ is a family of balls all containing some point and no ball contains the center of another ball, then the cardinality of $\mathcal{F}$ is no more than $J(d)$.

It remains to generalize the general position argument for the Euclidean balls used in the proof of theorem 1.1. We say a family $\mathcal{U}$ of bounded, open, convex sets in $\mathbb{R}^{d}$ is in general position if for any $d$ of the sets in the family, $U_{1}, \ldots, U_{d}$, we have that $\partial\left(U_{1}\right) \cap \ldots \cap \partial\left(U_{d}\right)$ is finite. We say that $x \in \mathbb{R}^{d}$ is a multiple point for $\mathcal{U}$ if for some $U_{1}, \ldots, U_{d} \in \mathcal{U}$ we have $x \in \partial\left(U_{1}\right) \cap \ldots \cap \partial\left(U_{d}\right)$. Thus, if $\mathcal{U}$ is finite and in general position, there are only finitely many multiple points for $\mathcal{U}$. Also as before, we say the multiplicity of $x \in \mathbb{R}^{d}$ with respect to $\mathcal{U}$ is the cardinality of $U \in \mathcal{U}$ such that $x \in \partial(U)$.

We claim it suffices to prove the following theorem.

Theorem 3.2. Let $U_{1}, \ldots, U_{d}$ be bounded, open, convex sets in $\mathbb{R}^{d}$. Then for Lebesgue measure almost all $v_{1}, \ldots, v_{d} \in \mathbb{R}^{d}$, the sets $\tilde{U}_{i}=U_{i}+v_{i}, 1 \leq i \leq d$, are in general position. 
Assuming theorem 3.2, suppose the sub-families $\mathcal{N}_{j}^{i}$ for $1 \leq i \leq p, j \leq n$ have been defined and satisfy (1), (2), ( $\left.3^{\prime}\right),(4)$, and (5), where (4) now reads: the balls of $\mathcal{D}$ are in general position. Inductively, we assume also the family $\mathcal{F}=\mathcal{D}$ satisfies:

6. For all $1 \leq e<d$ and $B_{1}, \ldots, B_{e} \in \mathcal{F}$, and for any $r_{e+1}, \ldots, r_{d} \in \mathbb{Q}^{+}$, for Lebesgue measure almost all $x_{e+1}, \ldots, x_{d} \in \mathbb{R}^{d}$ we have that the $\rho$-balls $B_{1}, \ldots, B_{e}, B_{\rho}\left(x_{e+1}, r_{e+1}\right), \ldots, B_{\rho}\left(x_{d}, r_{d}\right)$ are in general position.

Construct the families $\mathcal{W}, \mathcal{V}$ as in the proof of theorem 1.1, and color them also as in that theorem. We may assume that each ball in $\mathcal{W} \cup \mathcal{V}$ is of the form $B_{\rho}(x, r)$ where $r \in \mathbb{Q}^{+}$. Thus, the balls in $\mathcal{W} \cup \mathcal{V}$ satisfy $(1),(2),\left(3^{\prime}\right)$, and we fix $\epsilon>0$ such that if we translate these balls each by a distance less than $\epsilon$, then the translated balls still satisfy these three properties. Let $E_{1}, \ldots, E_{k}$ enumerate the balls in $\mathcal{E} \doteq \mathcal{W} \cup \mathcal{V}$. We successively translate the $E_{i}$ (by distances $<\epsilon$ ) to new balls $E_{i}^{\prime}$ which satisfy also (4), (5), (6). Assume that $E_{1}^{\prime}, \ldots, E_{l}^{\prime}$ for some $l<k$ have been defined, and that $\mathcal{F}=\mathcal{D} \cup\left\{E_{1}^{\prime}, \ldots, E_{l}^{\prime}\right\}$ satisfies (4), (5), (6). Let $E_{l+1}=B_{\rho}\left(x_{l+1}, r_{l+1}\right)$, so $r_{l+1} \in \mathbb{Q}^{+}$. ¿From theorem 3.2, Fubini's theorem, and the countable additivity of Lebesgue measure, it follows that for almost all $y_{l+1} \in \mathbb{R}^{d}$, and for almost all $z_{l+2}, \ldots, z_{l+d} \in \mathbb{R}^{d}$ and any $s_{l+2}, \ldots, s_{l+d} \in \mathbb{Q}^{+}$, that $B_{\rho}\left(y_{l+1}, r_{l+1}\right), B_{\rho}\left(z_{l+2}, s_{l+2}\right), \ldots, B_{\rho}\left(z_{l+d}, s_{l+d}\right)$ are in general position. Again by Fubini, for almost all $y_{l+1}$, and any $e<d-1, B_{1}, \ldots, B_{e} \in \mathcal{D} \cup\left\{E_{1}^{\prime}, \ldots, E_{l}^{\prime}\right\}$, and $s_{e+2}, \ldots, s_{d} \in \mathbb{Q}^{+}$, for almost all $z_{e+2}, \ldots, z_{d} \in \mathbb{R}^{d}$ we have that $B_{1}, \ldots, B_{e}$, $B_{\rho}\left(y_{l+1}, r_{l+1}\right), B_{\rho}\left(z_{e+2}, s_{e+2}\right), \ldots, B_{\rho}\left(z_{d}, s_{d}\right)$ are in general position. Also, for almost all $y_{l+1}$ and any $B_{1}, \ldots, B_{d-1} \in \mathcal{D} \cup\left\{E_{1}^{\prime}, \ldots, E_{l}^{\prime}\right\}$, the balls $B_{1}, \ldots, B_{d-1}$, $B_{\rho}\left(y_{l+1}, r_{l+1}\right)$ are in general position. Finally, since $\mathcal{D} \cup\left\{E_{1}^{\prime}, \ldots, E_{l}^{\prime}\right\}$ are in general position and $\partial\left(E_{l+1}\right)$ has Lebesgue (i.e. $\left.\mathcal{H}^{d}\right)$ measure zero, for almost all $y_{l+1}$, and any $B_{1}, \ldots, B_{d} \in \mathcal{D} \cup\left\{E_{1}^{\prime}, \ldots, E_{l}^{\prime}\right\}$, we have $\partial\left(B_{\rho}\left(y_{l+1}, r_{l+1}\right)\right) \cap \partial\left(B_{1}\right) \cap \cdots \cap \partial\left(B_{d}\right)=$ $\emptyset$. If we choose $y_{l+1}$ to lie in the complement of the measure zero sets just described, and with $y_{l+1}$ less than $\epsilon$ from $x_{l+1}$ in Euclidean distance, and set $E_{l+1}^{\prime}=B_{\rho}\left(y_{l+1}, r_{l+1}\right)$, then the balls $\mathcal{D} \cup\left\{E_{1}^{\prime}, \ldots, E_{l+1}^{\prime}\right\}$ satisfy (4), (5), and (6). This completes the proof of theorem 1.1.

It remains to prove theorem 3.2 .

Definition 3.1. A Borel set $A \subseteq \mathbb{R}^{d}$ has the $s$-dimensional lower density property if $\exists c, \delta>0 \forall x \in A \forall 0<r<\delta\left[\overline{\mathcal{H}}^{s}(A \cap B(x, r))>c r^{s}\right]$.

The following elementary lemma is well-known, and included for the sake of completeness.

Lemma 3.1. If $U \subseteq \mathbb{R}^{d}$ is a bounded, open, convex set, then $\partial(U)$ has the $d-1$ dimensional lower density property.

Proof. Without loss of generality we may assume $\mathbf{0} \in U$. Fix $R_{1}, R_{2}>0$ such that $B\left(\mathbf{0}, R_{1}\right) \subseteq U \subseteq B\left(\mathbf{0}, R_{2}\right)$. For any $x \neq \mathbf{0}$ in $\mathbb{R}^{d}$, let $N_{x}$ be the $d-1$ dimensional plane through $x$ and normal to the line containing $\mathbf{0}$ and $x$. Note that if $x \in \partial(U)$ then the interior of the "cone" $C_{x}$ which is the convex hull of $\{x\}$ and $B\left(\mathbf{0}, R_{1}\right)$ is contained in $U$. Thus there is a $\theta_{0}<\pi / 2$ such that if $x \in \partial(U)$ and $T_{x}$ is a support hyperplane for $U$ containing $x$, then the angle between $N_{x}$ and $T_{x}$ is $<\theta_{0}$. For $R_{1}<|x|<R_{2}$, let $C_{x}^{\prime}$ be the convex hull of $\{x\}$ and $B\left(\mathbf{0}, R_{1} / 2\right)$.

Let $\delta>0$ be sufficiently small so that the following properties are satisfied. First, suppose $|x|>R_{1}$ and $r<\delta$. Let $B=B(x, r)$, and let $D=B \cap C_{x}^{\prime}$. Then for any $y \in D$, the portion of the line $L_{y}$ containing $\mathbf{0}$ and $y$ between $y$ and $N$ is contained within $B$. Second, for any $y \in B$ and any support hyperplane $T_{x}$ for $U$ through $x$, 
the angle between $L_{y}$ and $T_{x}$ is greater than $\theta_{1}$, for some fixed $\theta_{1}>0$. That this is possible follows from $\theta_{0}<\pi / 2$.

To see this works, fix $x \in \partial(U)$, and $r<\delta$, and let $B=B(x, r)$. Let $D=D_{x}$ be the disk $D_{x}=B \cap N$, where $N$ is the hyperplane (parallel to $N_{x}$ ) containing $\partial(B) \cap C_{x}^{\prime}$. Let $T_{x}$ be a support hyperplane for $U$ through $x$. Note that $T_{x} \cap C_{x}^{\prime}=\emptyset$. For any $y \in D$, if the line $L_{y}$ from 0 to $y$ intersects $T_{x}$ before $N_{x}$ (not necessarily strictly before), then $L_{y} \cap B$ contains a point of $\partial(U)$. Let $E=E_{x} \subseteq D$ be the set of $y$ for which this happens. Thus, $\mathcal{H}^{d-1}(E) \geq(1 / 2) \mathcal{H}^{d-1}(D)$. For $y \in E$, let $p_{y}$ be the point $p_{y}=L_{y} \cap \partial(U)$, and let $q_{y}=L_{y} \cap T_{x}$. For any $y \in E$, the angle $\theta(y)$ between $L_{y}$ and $T_{x}$ is bounded below by $\theta_{1}>0$. It follows that there is a constant $C>0$, which is independent of $x$, such that for all $y_{1}, y_{2} \in E,\left|q_{y_{1}}-q_{y_{2}}\right| \leq C\left|p_{y_{1}}-p_{y_{2}}\right|$, that is, the map $p_{y} \rightarrow q_{y}$ is uniformly Lipschitz with constant $C$. Let $E^{\prime}=\left\{p_{y}: y \in E\right\}$, and $E^{\prime \prime}=\left\{q_{y}: y \in E\right\}$. Thus, $\mathcal{H}^{d-1}\left(E^{\prime \prime}\right) \leq C^{d-1} \mathcal{H}^{d-1}\left(E^{\prime}\right)$. But clearly there is a constant $D>0$ independent of $x$ such that $\mathcal{H}^{d-1}\left(E^{\prime \prime}\right) \geq D \mathcal{H}^{d-1}(E)$. Thus,

$$
\mathcal{H}^{d-1}(B \cap \partial(U)) \geq \mathcal{H}^{d-1}\left(E^{\prime}\right) \geq \frac{\mathcal{H}^{d-1}\left(E^{\prime \prime}\right)}{C^{d-1}} \geq \frac{D}{C^{d-1}} \mathcal{H}^{d-1}(E) .
$$

Since the vertex angles of the cones $C_{x}^{\prime}$ are bounded away from 0 , there is a constant $\alpha>0$ such that $\mathcal{H}^{d-1}\left(D_{x}\right) \geq \alpha \mathcal{H}^{d-1}(\partial(B))$ for all $x \in \partial(U)$ and $B=B(x, r)$ where $r<\delta$. Hence,

$$
\mathcal{H}^{d-1}(B \cap \partial(U)) \geq \frac{\alpha D}{2 C^{d-1}} \mathcal{H}^{d-1}(\partial(B)) \geq c r^{d-1}
$$

for some constant $c>0$ independent of $x$.

We recall also the well-known fact that if $U$ is a bounded, open, convex set in $\mathbb{R}^{d}$, then $\mathcal{H}^{d-1}(\partial(U))$ is finite (in the notation of the previous proof, the map that sends $y \in \partial\left(B\left(\mathbf{0}, R_{1}\right)\right)$ to $L_{y} \cap \partial(U)$ is Lipschitz). To prove theorem 3.2, it suffices to prove the following more general result (recall $\mathcal{H}^{0}$ is just counting measure).

Theorem 3.3. Let $A_{1}, \ldots, A_{i} \subseteq \mathbb{R}^{d}$ be Borel sets with $\mathcal{H}^{d-1}\left(A_{j}\right)<\infty$ for $1 \leq j \leq$ $i$, and such that each $A_{j}$ has the $d-1$ dimensional lower density property. Then for Lebesgue almost all $v_{1}, \ldots, v_{i} \in \mathbb{R}^{d}, \mathcal{H}^{d-i}\left(\tau_{v_{1}}\left(A_{1}\right) \cap \cdots \cap \tau_{v_{i}}\left(A_{i}\right)\right)<\infty$, where $\tau_{v}(A) \doteq A+v$.

In order to prove theorem 3.3, we require the following lemma which is essentially theorem 2 of [2] (though stated in a slightly different form).

Lemma 3.2. Let $A, B \subseteq \mathbb{R}^{d}$ be Borel sets with $\mathcal{H}^{s}(A)<\infty, \mathcal{H}^{t}(B)<\infty$. Suppose $A$ has the $s$-dimensional lower density property. Then $\mathcal{H}^{s+t}(A \times B)<\infty$.

Proof. By the lower density property, fix $c>0$ and $r_{0}>0$ such that $\forall x \in A \forall r<r_{0}$ $\left[\mathcal{H}^{s}(A \cap B(x, r)) \geq c r^{s}\right]$. Let $0<\epsilon<r_{0}$, and $\delta>0$. Let $\mathcal{D}$ be an $\epsilon$-mesh cover of $B$ such that $\sum_{D \in \mathcal{D}}|D|^{t}<\mathcal{H}^{t}(B)+\delta$, where $|D|$ denotes the diameter of $D$. For each $D \in \mathcal{D}$, let $\mathcal{C}^{D}=\{B(x, r): x \in A, r=|D| / 2\}$. By Besicovitch's covering theorem, let $\mathcal{C}_{1}^{D}, \ldots, \mathcal{C}_{b(d)}^{D}$ be subfamilies of $\mathcal{C}$ with $A \subseteq \bigcup_{i=1}^{b(d)} \cup \mathcal{C}_{i}^{D}$ and such that the balls in each subfamily $\mathcal{C}_{i}^{D}$ are pairwise disjoint. Since

$$
\mathcal{H}^{s}(A) \geq \sum_{B(x, r) \in \mathcal{C}_{i}^{D}} \mathcal{H}^{s}(A \cap B(x, r)) \geq \operatorname{card}\left(\mathcal{C}_{i}^{D}\right) \cdot c r^{s},
$$


it follows that $\operatorname{card}\left(\mathcal{C}_{i}^{D}\right) \leq \frac{\mathcal{H}^{s}(A)}{c r^{s}}$ for each $i$. Let $\mathcal{W}=\bigcup_{D \in \mathcal{D}} \bigcup_{i=1}^{b(d)} \bigcup_{C \in \mathcal{C}_{i}^{D}} C \times D$ Then $\mathcal{W}$ is a $\sqrt{2} \epsilon$ mesh cover of $A \times B$. Also,

$$
\begin{aligned}
\sum_{D \in \mathcal{D}} \sum_{i=1}^{b(d)} \sum_{C \in \mathcal{C}_{i}^{D}}|C \times D|^{s+t} & \leq 2^{(s+t) / 2} \sum_{D \in \mathcal{D}} \sum_{i=1}^{b(d)} \sum_{C \in \mathcal{C}_{i}^{D}}|D|^{s+t} \\
& \leq 2^{(s+t) / 2} \sum_{D \in \mathcal{D}}|D|^{s+t} \sum_{i=1}^{b(d)} \operatorname{card}\left(\mathcal{C}_{i}^{D}\right) \\
& \leq 2^{(s+t) / 2} \sum_{D \in \mathcal{D}}|D|^{s+t} \frac{b(d) \mathcal{H}^{s}(A)}{|D|^{s}} \\
& \leq 2^{(s+t) / 2} b(d) \mathcal{H}^{s}(A)\left(\mathcal{H}^{t}(B)+\delta\right) .
\end{aligned}
$$

Letting $\delta$ go to 0 , we have

$$
\mathcal{H}_{\sqrt{2} \epsilon}^{s+t}(A \times B) \leq 2^{(s+t) / 2} b(d) \mathcal{H}^{s}(A) \mathcal{H}^{t}(B)
$$

Letting $\epsilon$ go to 0 , we have $\mathcal{H}^{s+t}(A \times B) \leq 2^{(s+t) / 2} b(d) \mathcal{H}^{s}(A) \mathcal{H}^{t}(B)$.

We now prove theorem 3.3 by induction on $i$. For $i=1$ the statement is trivial. Assume now the theorem holds for $i<d$, and we verify it for $i+1$. By Fubini, it suffices to fix $v_{1}, \ldots, v_{i} \in \mathbb{R}^{d}$ such that $\mathcal{H}^{d-i}\left(\tau_{v_{1}}\left(A_{1}\right) \cap \cdots \cap \tau_{v_{i}}\left(A_{i}\right)\right)<\infty$, and show that for Lebesgue almost all $v_{i+1} \in \mathbb{R}^{d}$ that $\mathcal{H}^{d-i-1}\left(\tau_{v_{1}}\left(A_{1}\right) \cap \cdots \cap \tau_{v_{i}}\left(A_{i}\right) \cap\right.$ $\left.\tau_{v_{i+1}}\left(A_{i+1}\right)\right)<\infty$.

Theorem 7.7 of [4] states that if $A \subseteq \mathbb{R}^{n}, f: A \rightarrow \mathbb{R}^{m}$ is a Lipschitz map, and $m \leq s \leq n$, then

$$
\int^{*} \mathcal{H}^{s-m}\left(A \cap f^{-1}\{y\}\right) d \mathcal{L}^{m} y \leq 2^{m} \alpha(m) \operatorname{Lip}(f)^{m} \mathcal{H}^{s}(A),
$$

where $\int^{*}$ denotes the upper integral, and $\alpha(m)$ is a constant depending only on $m$ (the $m$-dimensional measure of the unit ball in $\mathbb{R}^{m}$ ). Let $C=\tau_{v_{1}}\left(A_{1}\right) \cap \cdots \cap \tau_{v_{i}}\left(A_{i}\right)$, so $\mathcal{H}^{d-i}(C)<\infty$. Consider the map $S: \mathbb{R}^{d} \times \mathbb{R}^{d} \rightarrow \mathbb{R}^{d}$ given by $S(x, y)=x-y$. Clearly $S$ is Lipschitz. ¿From lemma 3.2, $\mathcal{H}^{2 d-i-1}\left(C \times A_{i+1}\right)<\infty$. Applying the above formula with $f=S, n=2 d, m=d$, and $s=2 d-i-1 \geq m$, we have

$$
\int^{*} \mathcal{H}^{d-i-1}\left(\left(C \times A_{i+1}\right) \cap S^{-1}\{y\}\right) d \mathcal{L}^{d} y \leq c \mathcal{H}^{2 d-i-1}\left(C \times A_{i+1}\right)<\infty .
$$

In particular, for almost all $y \in \mathbb{R}^{d}$ we must have that $\mathcal{H}^{d-i-1}\left(E_{y}\right)$ is finite, where $E_{y}=\left(C \times A_{i+1}\right) \cap S^{-1}\{y\}$. Now, $E_{y}=\left\{(x, x-y): x \in C, x-y \in A_{i+1}\right\}$. Since $\mathcal{H}^{d-i-1}$ is translation invariant, $\mathcal{H}^{d-i-1}\left(E_{y}\right)=\mathcal{H}^{d-i-1}\left(C \cap \tau_{y}\left(A_{i+1}\right)\right)<\infty$. Writing $v_{i+1}=y$, this verifies theorem 3.3 for $i+1$. This finishes the proof of theorem 3.3, and hence of theorem 1.1.

\section{References}

[1] A. S. Besicovitch, A general form of the covering principle and relative differentiation of additive functions, Proc. Cambridge Phil. Soc. 41 (1945), 103-110.

[2] A. S. Besicovitch and A. P. Moran, The measure of product and cylinder sets, Journal of the London Math. Soc. 20 (1945), 110-120.

[3] R. O. Davies, Measures not approximable or not specifiable by means of balls, Mathematika 18 (1971), 157-160.

[4] P. Mattila, Geometry of sets and measures in Euclidean spaces, Cambridge University Press, 1995 
[5] A. P. Morse, Perfect blankets, Trans. Amer. Math. Soc. 61 (1947), 418-442.

[6] T. Neubrunn, A note on quantum probability spaces, Proc. Amer. Math. Soc. 25 (1970), 672-675.

[7] V. Olejček, Generation of a q- $\sigma$-algebra in the plane. Proc. Conf. Topology and Measure V, Wissenshaftliche Beitrage der Ernst-Moritz-Arndt-Universitat Greifswald, 1988, 121-125.

[8] V. Olejček, The $\sigma$-class generated by balls contains all Borel sets, Proc. Amer. Math. Soc. 123 (1995), 3665-3675.

[9] D. Preiss and J. Tiser, Measures in Banach spaces are determined by their values on balls, Mathematika 38 (1991), 391-397.

Department of Mathematics, University of North Texas, Denton, TX 76203-5116

E-mail address: jackson@unt.edu

Department of Mathematics, University of North Texas, Denton, Texas 76203-5116

E-mail address: mauldin@dynamics.math.unt.edu 